\title{
PinMol: Python application for designing molecular beacons for live cell imaging of endogenous mRNAs
}

\author{
LIVIA V. BAYER, ${ }^{1,2}$ OMAR S. OMAR, ${ }^{1,2}$ DIANA P. BRATU, ${ }^{1,2}$ and IRINA E. CATRINA ${ }^{1}$ \\ ${ }^{1}$ Biological Sciences Department, Hunter College, City University of New York, New York, New York 10065, USA \\ ${ }^{2}$ Program in Molecular, Cellular, and Developmental Biology, The Graduate Center, City University of New York, New York, New York 10016, USA
}

\begin{abstract}
Molecular beacons are nucleic acid oligomers labeled with a fluorophore and a quencher that fold in a hairpin-shaped structure, which fluoresce only when bound to their target RNA. They are used for the visualization of endogenous mRNAs in live cells. Here, we report a Python program (PinMol) that designs molecular beacons best suited for live cell imaging by using structural information from secondary structures of the target RNA, predicted via energy minimization approaches. PinMol takes into account the accessibility of the targeted regions, as well as the inter- and intramolecular interactions of each selected probe. To demonstrate its applicability, we synthesized an oskar mRNA-specific molecular beacon (osk1236), which is selected by PinMol to target a more accessible region than a manually designed oskar-specific molecular beacon (osk2216). We previously demonstrated osk2216 to be efficient in detecting oskar mRNA in in vivo experiments. Here, we show that osk1236 outperformed osk2216 in live cell imaging experiments.
\end{abstract}

Keywords: molecular beacon; mRNA detection; RNA secondary structure; live cell imaging; nucleic acid hybridization

\section{INTRODUCTION}

Currently, there are a few methods that are used to image RNA in live tissues and cells, and each has its advantages and disadvantages (Weil et al. 2010). We use the molecular beacon technology for live cell imaging of mRNA transport and localization in the fruit fly egg chamber (Bratu et al. 2003). Molecular beacons are oligonucleotides labeled with a fluorophore at the $5^{\prime}$ end and a quencher at the $3^{\prime}$ end that are designed to fold into a hairpin shape (Fig. 1). In this study, we use the terms "probe" and "stem" to refer to the loop or single-stranded region of the hairpin with a sequence complementary to the mRNA target (18-26 nucleotides [nt]), and to the double-stranded region (five G/C-rich base pairs), respectively. In the presence of target, the hybridization of the probe region with the target is energetically more favorable than preserving the duplex in the stem, which leads to the separation of the fluorophore/quencher pair and a significant increase in the fluorescence signal (Fig. 1). One major advantage of using this technology is that molecular beacons allow direct visualization of endogenous mRNAs. However, not many groups have reported in vivo mRNA imaging using molecular beacons. One reason is the difficulty in finding an efficient delivery method for the chosen live specimen. We have successfully introduced nuclease-resistant molecular

Corresponding author: icatrina@hunter.cuny.edu

Article is online at http://www.rnajournal.org/cgi/doi/10.1261/rna. 069542.118 . beacons in fruit fly egg chambers via microinjection, to study transport and localization of endogenous maternal mRNAs (Bratu et al. 2003). Others have described the use of toxin-based membrane permeabilization and microporation for efficient cytoplasmic delivery of molecular beacons (Chen et al. 2011), and more recently, gold nanoparticles were shown to efficiently deliver molecular beacons into live breast cancer cells for multiplex detection of mRNAs (Jackson et al. 2016). Another reason for the limited in vivo use of molecular beacons is the challenge to design them, as several properties need to be optimized to ensure specific and efficient detection of the target RNA. Here, we describe a new open-source Python-based application that allows fast and easy prediction of molecular beacons tailored for live cell imaging.

There are several stand-alone or server-based databases (e.g., miRBase [Griffiths-Jones et al. 2008]) and applications (e.g., TargetScan [Lewis et al. 2005], OligoWalk [Lu and Mathews 2008b], siRNA Wizard from InvivoGen) for the analysis and identification of targets of small RNAs, such as microRNAs (miRNA) and small interfering RNAs (siRNA), and for the design of small probes that efficiently bind to mRNA targets. However, freely available programs

(C) 2019 Bayer et al. This article is distributed exclusively by the RNA Society for the first 12 months after the full-issue publication date (see http:///rnajournal.cshlp.org/site/misc/terms.xhtml). After 12 months, it is available under a Creative Commons License (Attribution-NonCommercial 4.0 International), as described at http://creativecommons.org/licenses/by-nc/4.0/. 


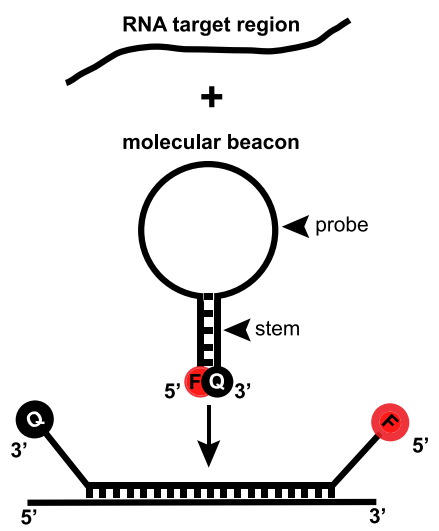

FIGURE 1. Molecular beacons, principle of operation. F, fluorophore, $\mathrm{Q}$, quencher.

for designing molecular beacons focus on their application for quantitative PCR (qPCR) primer design and analysis, and they usually limit the target region to less than 1200 bases (Table 1).

PinMol (Python program for designing hairpin-shaped molecular beacons) permits the fast design of molecular beacons suited for live cell imaging of any target mRNA in specimens amenable to oligonucleotide delivery and live cell imaging, by taking into consideration target accessibility to minimize false negative results, and cross homology to avoid false-positive results.

\section{RESULTS}

\section{The PinMol algorithm}

\section{Input file (Fig. 2: \#2, "ss-count file input")}

To assess target-site accessibility, it is common to evaluate the predicted secondary structure of the RNA target of interest, which is generated using either phylogenetic analysis or free energy minimization algorithms. The efficiency of a candidate probe is directly correlated to the accuracy of the predicted secondary structure of the target RNA. When using results from free energy minimization algorithms, it is recommended to consider the global structure of a target RNA rather than a local structure prediction of a limited region within that target (Lu and Mathews 2008a). mfold, a software commonly used to predict the secondary structure of nucleic acids using experimentally derived thermodynamic parameters, yields a minimum free energy (MFE) structure and a user-selected number of suboptimal (SO) structures (Zuker 2003). mfold, RNAstructure, and the Vienna RNAfold package use similar algorithms, and although they use different sets of RNA thermodynamic parameters (mfold: 1999 Turner set; RNAstructure: 2004 Turner set; Vienna RNAfold: choice of 1999 or subset of 2004 Turner set, 2007 Andronescu set) that result in slightly different predictions, their accuracy in performance are comparable. mfold and RNAstructure predict MFE and suboptimal RNA secondary structures, while the RNAfold generates an MFE secondary structure (Zuker 2003; Reuter and Mathews 2010; Lorenz et al. 2011). PinMol uses as input the "ss-count" file generated by mfold upon folding the RNA target sequence of interest. This file lists, for each nucleotide in the target RNA, the number of structures out of the total number predicted (comprising the MFE and suboptimal structures) in which the corresponding nucleotide is single-stranded.

\section{User-defined parameters}

1. Probe length (Fig. 2: \#2, "probe length"); sequence complementary to the RNA target site, a value between 18 and 26 nt.

2. Number of desired molecular beacons to be designed (Fig. 2: \#8, "number of probes"); between 2 and 50, or maximum number of probes that meet the design criteria, whichever is smaller.

3. Target region (optional) for molecular beacon design (Fig. 2: \#2, "target region"), while still considering the predicted global structure. This is useful when there are certain stretches within the mRNA target that are known to be subject to regulation in the cell or tissue of interest (e.g., regions that interact with protein factors), which may render them inaccessible for probe hybridization, and thus should not be taken into account for probe design.

4. Cross homology with other RNA targets (optional) (Fig. 2: \#9 to \#11, "perform BLAST?"); this analysis is performed using a user-generated, external "xml" file from the NCBI "blastn" application.

TABLE 1. Comparison of programs for designing molecular beacons, which take into consideration the structure of the RNA target

\begin{tabular}{|c|c|c|c|c|}
\hline Program & Open source & Suboptimal structures & Maximum target length (nt) & Recommended application \\
\hline PinMol & Yes & Yes & Up to 8000 & In vivo imaging \\
\hline OligoArchitect ${ }^{\mathrm{a}}$ & Yes & No & Up to 1200 & qPCR \\
\hline Other (BeaconDesigner, NYtor) & No & No & Up to 8000 & qPCR \\
\hline
\end{tabular}

(nt) Nucleotide.

aPowered by BeaconDesigner. 


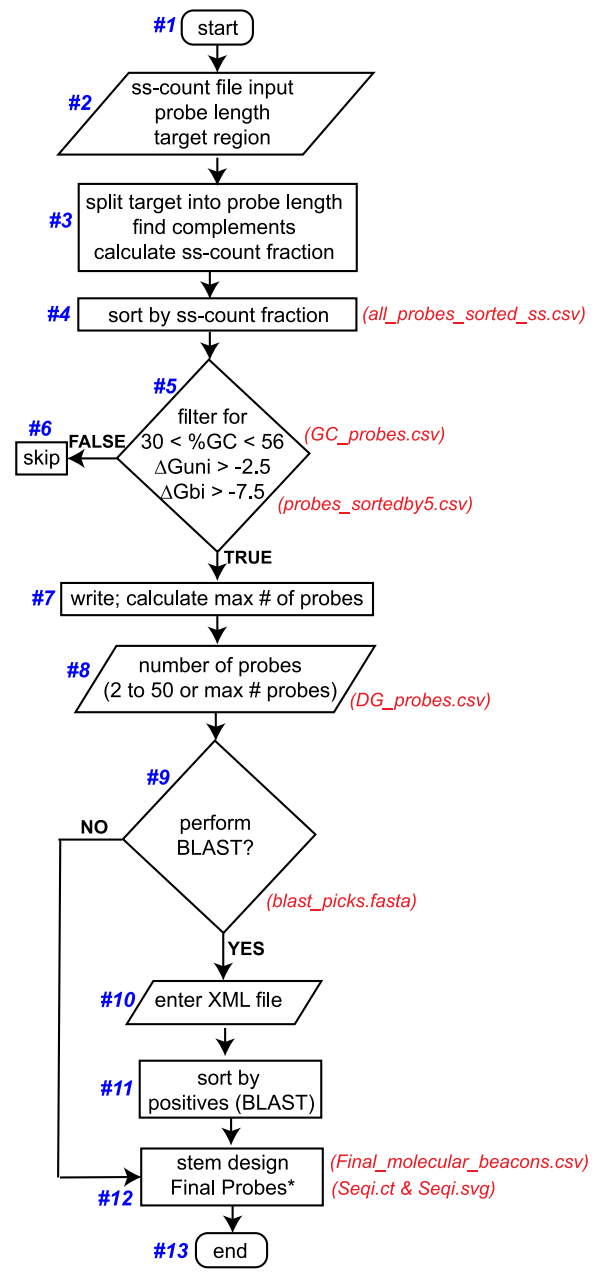

FIGURE 2. Simplified flow chart for the PinMol algorithm. Intermediate files (red) that are generated and saved by PinMol. $\Delta \mathrm{G}_{\text {uni, }}$, free energy of unimolecular folding or $\Delta \mathrm{G}_{\text {unimoli }} \Delta \mathrm{G}_{\mathrm{bi}}$, free energy of bimolecular folding or $\Delta \mathrm{G}_{\text {bimol. }}\left(^{*}\right)$ Molecular beacons that are predicted to fold in a highly structured MFE structure $(<-7.2 \mathrm{kcal} /$ $\mathrm{mol}$ ), or that fail to fold into a hairpin shape, are eliminated. All output files, generated by PinMo/when designing osk-specific molecular beacons with 24-nt-long probe region, are available for download on our laboratory's webpage (https://bratulab.wordpress.com/software/).

\section{PinMol probe ranking and selection}

Initially, all possible probes for the user-selected probe length are sorted according to a target "ss-count fraction" (Fig. 2: \#4, "sort by ss-count fraction," "all_probes_ sorted_ss.csv"). We define this fraction as the ratio of the sum of the ss-count of each nucleotide [ss-count $\left(n t_{i}\right)$ ] for a selected probe length to the product of the probe length (I) and the number of secondary structures included in the input file $\left(n_{\text {str }}\right)$ (Fig. 2: \#3, "calculate ss-count fraction") (Equation 1).

$$
f_{\text {ss-count }}=\frac{\sum_{1}^{l} s s-\operatorname{count}\left(n t_{i}\right)}{l \times n_{\text {str }}} .
$$

Therefore, $f_{\text {ss-count }}$ is equal to one for a fully singlestranded target region, and zero for a fully double-stranded target region. From these sorted probes, the program filters out the probes with a percentage of GC nucleotides smaller than 31 or greater than 55 (Fig. 2: \#6, "skip"; Bratu et al. 2011). For the probes that meet these requirements (Fig. 2: \#5, "filter for $30<\% G C<56$ "), PinMol calculates the free energy of unimolecular $\left(\Delta \mathrm{G}_{\text {unimol }}\right)$ and bimolecular $\left(\Delta \mathrm{G}_{\text {bimol }}\right)$ folding using the oligoscreen subroutine of the RNAstructure text interface application (Mathews 2006; Reuter and Mathews 2010).

Molecular beacons used in live cell imaging experiments are often synthesized with a 2'-O-methyl RNA (2'OMe) modified backbone to ensure resistance to cellular nucleases. Therefore, we used RNA parameters to calculate folding free energies, as they more closely match 2'OMe/ RNA hybridization profiles when compared to DNA parameters (Tsourkas et al. 2002). To find probes with maximum efficiency for target detection, PinMol filters out probes that have a $\Delta \mathrm{G}_{\text {unimol }} \leq-2.5 \mathrm{kcal} / \mathrm{mol}$ and $\Delta \mathrm{G}_{\text {bimol }} \leq-7.5$ $\mathrm{kcal} / \mathrm{mol}$ (Fig. 2: \#6, "skip"), in order to minimize probe-target hybridization constraints that might be imposed by a highly structured probe region (Fig. 2: \#5, " $\Delta$ Guni > -2.5 and $\Delta \mathrm{Gbi}>-7.5^{\prime \prime}$ ).

\section{Stem design (Fig. 2: \#12, "stem design/Final Probes")}

After the probes have been selected, the stem is designed to form five G/C-rich base pairs. The program takes into consideration if any of the end nucleotides of the selected probe sequence are complementary to each other (up to three on each end), in which case the stem is adjusted to incorporate them. This means that when such complementarity is found, the probe region that is single-stranded may be shorter than the originally selected probe length.

The PinMol stem design function also searches for possible complementarity of the newly added stem sequence with the selected probe sequence. When such complementarity is found, the stem sequence is redesigned to ensure that the molecular beacon will fold into a hairpin shape. After the stem is added, each probe is folded and the MFE structure is drawn using the fold and draw subroutines of RNAstructure, respectively (Mathews 2006; Reuter and Mathews 2010). Highly structured molecular beacons are discarded by filtering out the structures with a folding MFE $\leq-7.2 \mathrm{kcal} / \mathrm{mol}$ (Fig. 2). In addition, a few molecular beacons that do not fold in the desired hairpin conformation can be obtained, especially for shorter probe lengths. Therefore, after the above described filtering steps, PinMol also filters out probes for which the first and the last nucleotides of the molecular beacon sequence are not basepaired in the MFE structure listed in the corresponding "ct" output files generated by the fold subroutine (Fig. 2: \#12, "Final Probes," "Final_molecular_beacons.csv," "Seqi.ct," and "Seqi.svg"). 
Although PinMol does not include the selection of the fluorophore/quencher pair, when designing molecular beacons it should also be taken into consideration that some pairs have a stabilizing effect (e.g., for the same molecular beacon sequence $\mathrm{Cy} 5 / \mathrm{BHO}-2$ increases the melting temperature [Tm] by $5^{\circ} \mathrm{C}$ as compared to $\mathrm{Cy} 5 /$ Dabcyl) (Marras et al. 2002).

\section{Defining an optimum number of selected probes}

To define an optimum number of probes without limiting the $f_{\text {ss-count, }}$ we determined the number of probes that have a $f_{\text {ss-count }}>0.5$ for oskar (osk) mRNA (Fig. 3A), a maternal mRNA that we have successfully visualized in live fruit fly egg chambers using molecular beacons (Bratu et al. 2003; Mhlanga et al. 2009; Catrina et al. 2016). We determined the $f_{\text {ss-count }}$ for all osk probes with a probe length ranging between 14 and 28 nt using target structural information from two different PinMol input files, which were obtained with mfold (MFE, 31STR). The maximum number of osk probes with a $f_{\text {ss-count }}>0.5$ was obtained for the 31STR input for a probe length of $14 \mathrm{nt}$ (725 probes). The minimum

A

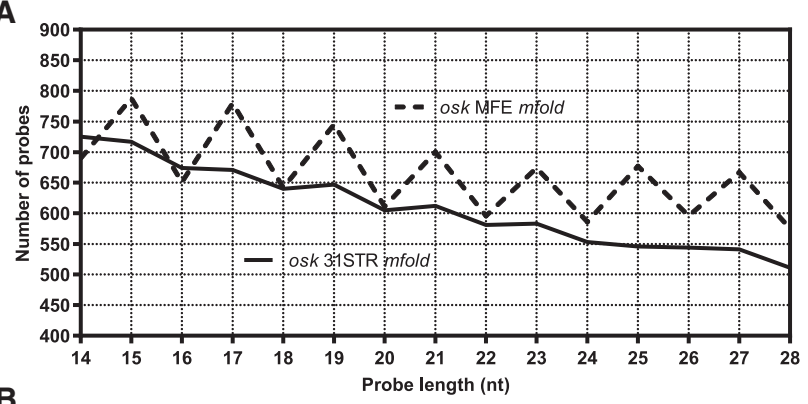

B

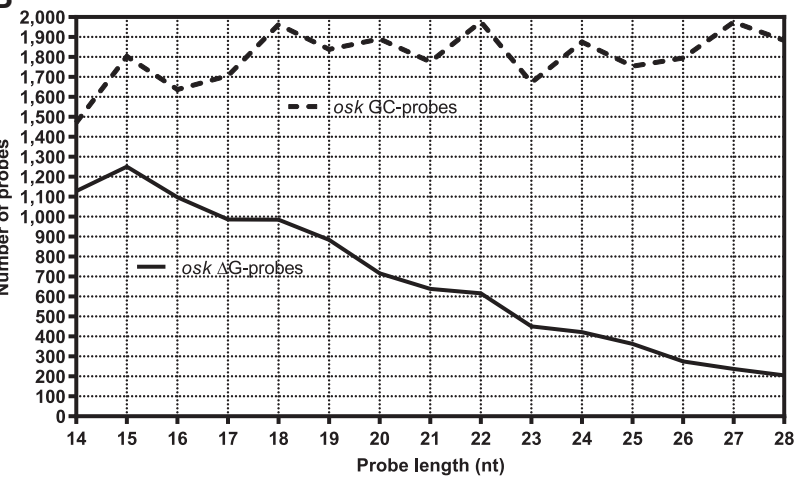

FIGURE 3. The number of probes generated using PinMol for osk mRNA target (2869 nt). (A) The number of probes of a given length (between 14 and $28 \mathrm{nt}$ ) that target a region with a $f_{\text {ss-count }}$ larger than 0.5 (more than half of the bases are single-stranded) when using osk mRNA mfold predicted secondary structures (MFE—-dashed line; 31STR-solid line). (B) The number of probes, for a given probe length, that meet the GC-content requirement (equal to, or between $31 \%$ and $55 \%$ ) (GC-probes-dashed line), and number of probes that meet the GC-content requirement and the $\Delta \mathrm{G}_{\text {unimol }}$ and $\Delta \mathrm{G}_{\text {bimol }}$ limits $(>-2.5 \mathrm{and}>-7.5 \mathrm{kcal} / \mathrm{mol}$, respectively, $\Delta \mathrm{G}$-probes—solid line) for osk mRNA. number of osk probes with a $f_{\text {ss-count }}>0.5$ was obtained for the 31STR input for a probe length of $28 \mathrm{nt}$ (511 probes). We found that limiting the number of probes to 50 , although it may reduce the number of unique probes, ensures a high $f_{\text {ss-count }}$ for target RNAs of an average length (e.g., $2200 \mathrm{nt}$ for mammalian mRNAs). For shorter RNA targets, we recommend that the $f_{\text {ss-count }}$ corresponding to the regions targeted by the selected molecular beacons be evaluated on a case-by-case basis (Fig. 2: \#5, "probes_ sortedby5.csv" output file).

Next, we determined the number of probes for osk mRNA that meet the GC-content (GC-probes) (Fig. 2: \#5, "GC_probes.csv" output file), or both the GC-content and the folding energy ( $\Delta \mathrm{G}$-probes) requirements (Fig. 2: \#5, "probes_sortedby5.csv" output file), as described above, for probe lengths between 14 and 28 nt (Fig. 3B), and we found that more than 50 probes meet all the design criteria, for each probe length. Since probes are not selected or eliminated based on their $f_{\text {ss-count, the number of }}$ probes that meet the GC-content requirement, or meet the GC-content and energetic requirements was independent of the number of structures taken into consideration in the input file (MFE or 31STR) used to run PinMol (Fig. 3A vs. 3B).

\section{Cross homology with other RNAs}

In microarray assays using 50 nt-long oligonucleotides, in order to avoid false-positive signals, the maximum allowed cross complementarity is 16 consecutive nucleotides ( $\mathrm{He}$ et al. 2005). From studies with mismatched molecular beacons, it was shown that the molecular beacon/target hybrids are destabilized even with a single nucleotide mismatch, usually placed in the center of the probe sequence (Tyagi et al. 1998; Chen et al. 2007). When considering that the maximum probe length allowed by PinMol is $26 \mathrm{nt}$, such overlap should be less than 13 consecutive nucleotides for molecular beacons of maximum probe length, and this requirement can be relaxed for molecular beacons with probe lengths shorter than $26 \mathrm{nt}$. Additional information, specific to the model system of interest, should also be taken into consideration. For example, when imaging transcripts expressed in the fruit fly ovary, any homology overlap with other mRNAs not expressed in this tissue can be disregarded. Although PinMol includes an optional ranking of the chosen probes using the "xml" output file from a BLAST alignment search, it does not take into account the cell/tissue expression levels of the target. The probe ranking using BLAST alignment results is performed after the desired number of probes are selected, thus these probes are re-sorted in ascending order of the maximum number of positives found for a complementary match between each of the selected probe sequences and an mRNA other than the target of interest. Therefore, this feature is convenient when designing probes targeting ubiquitously 
expressed mRNAs, but may not be needed if the target mRNA has tissue-restricted expression levels. PinMol generates a file containing a list of the selected number of probes in the "FASTA" format (Fig. 2: \#9, "blast_picks. fasta" output file), which can be uploaded in the NCBI BLAST form. For mRNA targets, "blastn" search is recommended, using the reference RNA sequences or refseq_rna database for the organism of interest and default parameters. For other RNA targets, the "blastn" search with the nucleotide collection (nr/nt) and default parameters should be used, as the program will prematurely terminate if it does not find at least one fully complementary match to each probe sequence.

PinMol provides ranks of the selected number of probes before and after BLAST alignment. Therefore, if BLAST is performed for the chosen probes, it is recommended that the target's tissue expression level for the corresponding organism is also taken into consideration, and that probes that are ranked based on cross homology results are carefully compared with the pre-BLAST ranking results. This comparison will identify candidate probes that rank highly before BLAST, but which are placed lower on the list after BLAST due to a cross homology with an mRNA that is not expressed in the tissue of interest.

\section{Comparison with experimental data}

Molecular beacons have been demonstrated to be highly specific probes, which are able to discriminate against a single nucleotide mismatch between a probe and a target region (Tyagi et al. 1998; Bratu et al. 2003). A false-positive signal resulting from a probe hybridizing to a different target is likely to occur in conditions where such mismatches are tolerated. These conditions are minimized in classical RNA-FISH (fluorescence in situ hybridization) experiments by using denaturing reagents and increasing the experimental temperature. However, for shorter probes, such as molecular beacons (18-26 nt), the penalty for mismatches greatly increases, and when combined with the energy requirement to open the stem of a hairpin, it allows for specific hybridization at physiological conditions, with target accessibility as the limiting factor.

\section{Molecular beacons specific to osk mRNA}

We have successfully visualized and analyzed osk mRNA transport and localization within the Drosophila melanogasteregg chamber using fluorescence confocal microscopy coupled with molecular beacons (Mhlanga et al. 2009). The $D$. melanogaster egg chamber goes through 14 stages of development, from germarium to stage 14 , and it is composed of somatic (follicle) and germline (one oocyte and 15 nurse cells) cells (McLaughlin and Bratu 2015). Several mRNAs have been reported to asymmetrically localize in the oocyte during oogenesis, and these processes are essential for the development and survival of the future embryo (Lasko 2012). osk mRNA is responsible for germplasm determination (Ephrussi et al. 1991), and it is one of the most studied mRNAs in fruit flies. It is actively transported from the nurse cells, and localized and anchored at the oocyte's posterior during mid- and late oogenesis (stages 7-9) (Ephrussi et al. 1991; Kim-Ha et al. 1991). Therefore, we selected osk mRNA as our target mRNA to compare the ranking of molecular beacons designed using PinMol with our previously manually designed molecular beacons (Bratu et al. 2003), using both in vitro and in vivo experiments.

After design, synthesis, and purification, the performance of a molecular beacon that is to be used in live cell imaging experiments is characterized by analyzing its (i) thermal denaturation profile and (ii) hybridization reaction with a complementary DNA oligonucleotide target and/or in vitro transcribed mRNA target. The thermal denaturation indicates whether the molecular beacon is properly folded into a hairpin shape, while the hybridization analysis estimates the binding efficiency of the probe to its target, as well as the fluorescence signal-to-background ratio (Bratu et al. 2011). The efficiency to hybridize with the target of molecular beacons that were manually designed to target several distinct regions within osk mRNA was determined using in vitro hybridization reactions with in vitro transcribed and folded full-length osk mRNA (Table 2; Bratu et al. 2003).

We compared the experimental ranking of these oskspecific molecular beacons with the ranking of PinMol designed molecular beacons for target structures predicted

TABLE 2. Ranking of osk mRNA-specific molecular beacons according to their performance in in vitro hybridization experiments using full-length osk RNA

\begin{tabular}{|c|c|c|c|c|c|}
\hline \multirow[b]{2}{*}{$\begin{array}{l}\text { Experimental } \\
\text { rank }\end{array}$} & \multirow[b]{2}{*}{$\begin{array}{c}\text { Base } \\
\text { number }\end{array}$} & \multirow[b]{2}{*}{$\begin{array}{l}\text { Probe } \\
\text { length }\end{array}$} & \multicolumn{3}{|c|}{ mfold/PinMol } \\
\hline & & & $\begin{array}{l}\text { MFE } \\
\text { rank }\end{array}$ & $\begin{array}{l}\text { 31STR } \\
\text { rank }\end{array}$ & $\begin{array}{l}31 \mathrm{STR} \\
f_{\text {ss-count }}\end{array}$ \\
\hline 1 & 2216 & 24 & 52 & 25 & 0.694 \\
\hline 2 & 967 & 23 & 32 & 54 & 0.616 \\
\hline 3 & 82 & 24 & 20 & 4 & 0.763 \\
\hline 4 & 358 & 21 & $\mathrm{n} / \mathrm{a}$ & $\mathrm{n} / \mathrm{a}$ & 0.548 \\
\hline 5 & 720 & 23 & $\mathrm{n} / \mathrm{a}$ & $\mathrm{n} / \mathrm{a}$ & 0.523 \\
\hline 6 & 2585 & 16 & 156 & 29 & 0.756 \\
\hline 7 & 1220 & 18 & 87 & 51 & 0.681 \\
\hline 8 & 544 & 21 & 146 & 200 & 0.512 \\
\hline 9 & 1853 & 22 & $\mathrm{n} / \mathrm{a}$ & $\mathrm{n} / \mathrm{a}$ & 0.516 \\
\hline 10 & 1667 & 23 & $\mathrm{n} / \mathrm{a}$ & $\mathrm{n} / \mathrm{a}$ & 0.642 \\
\hline 11 & 11 & 15 & $\mathrm{n} / \mathrm{a}$ & $\mathrm{n} / \mathrm{a}$ & 0.394 \\
\hline
\end{tabular}

(MFE) Minimum free energy structure. (31STR) MFE and 30 suboptimal structures. 
using mfold (Supplemental Table S1) when considering MFE or 31STR for osk mRNA. The ranking of the probes designed for 31STR were the closest to the experimental results, followed by MFE (Fig. 4). Several probes were chosen for the 1800-1900 nt region for MFE, which were found to perform poorly experimentally. However, when using 31STR mfold, no probes were selected for this region (Figs. 4 and 5A).

We also compared the top ten 24-nt-long probe sequences designed when using MFE or 31STR input files with previous experimental results, and found that probes selected for 31STR were from four different regions (10s, 1200s, 2200s, and 2800s) (Fig. 5B). Overall, our analysis suggests that the ranking of PinMol designed molecular beacons was better aligned with experimental data when $30 \mathrm{SO}$ structures were considered in addition to the MFE structure (Table 2; Fig. 4). Moreover, the PinMol rank of the top experimental probe improved when using 31STR versus MFE (Table 2; osk2216), while probes with poor experimental performance were eliminated during the filtering steps (Table 2; osk544, osk1853, and osk1667).

For detection of low abundance targets, it may be necessary to increase the number of fluorophores per RNA molecule by using multiple molecular beacons that bind to different regions along the target RNA. We found that the 50 designed probes were almost entirely encompassed within 10, strongly supported experimentally, and predicted to be accessible regions (each $100 \mathrm{nt}$ long) of osk mRNA (Fig. 4). This indicates that our program selected some "redundant" probes that showed differences of only a few nucleotides (e.g., osk2216 and osk2215). While it may be tempting to eliminate these redundant probes, we advise that this should not be done prior to performing BLAST alignment for determining cross homology with other mRNAs. Moreover, it should be considered that the $f_{\text {ss-count }}$ value decreases for probes ranked low in the initially filtered probe list (Fig. 2: \#8, "DG_probes.csv" PinMol output file). Although the stem design is limited to 50 total probes, PinMol saves the intermediate files containing all sorted probes after filtering (Fig. 2: \#5, "probes_sortedby5.cvs"), which can be used to further inspect the results, and if desired, to select smaller target regions, and then to redesign the probes.

As an example, we selected the region between 1301 and $1600 \mathrm{nt}$ of osk mRNA, for which 24 nt-long probes were not selected in the top 50 probes designed using PinMol (Fig. 5A). For this region, when using 31STR as input file the total number of possible probes that are $24 \mathrm{nt}$-long was 278, out of which 231 met the GC-content requirement, and of which 36 also met the folding energy requirements, but only 15 probes had a $f_{\text {ss-count }}>0.5$. The top choice before applying any filters was at base number 1381 with a $f_{\text {ss-count }}$ of only 0.547 , which was ranked 324 th in the list of all probes sorted in descending order by $f_{\text {ss-count, }}$ but osk1381 was eliminated during the initial filtering steps. The final top choice for this region was the probe at base number 1308 , with a $f_{\text {ss-count }}$ of 0.520 , and ranked 448th when considering all the probes sorted by PinMol for the full-length target osk mRNA. Our analysis showed that even the best molecular beacon that PinMol designed for this region targets a stretch that consists of single-stranded nucleotides for only approximately half of its length. This confirms that the selected target region was predicted to be highly structured, and therefore it is not suitable for targeting osk mRNA in both in vitro and in vivo experiments.

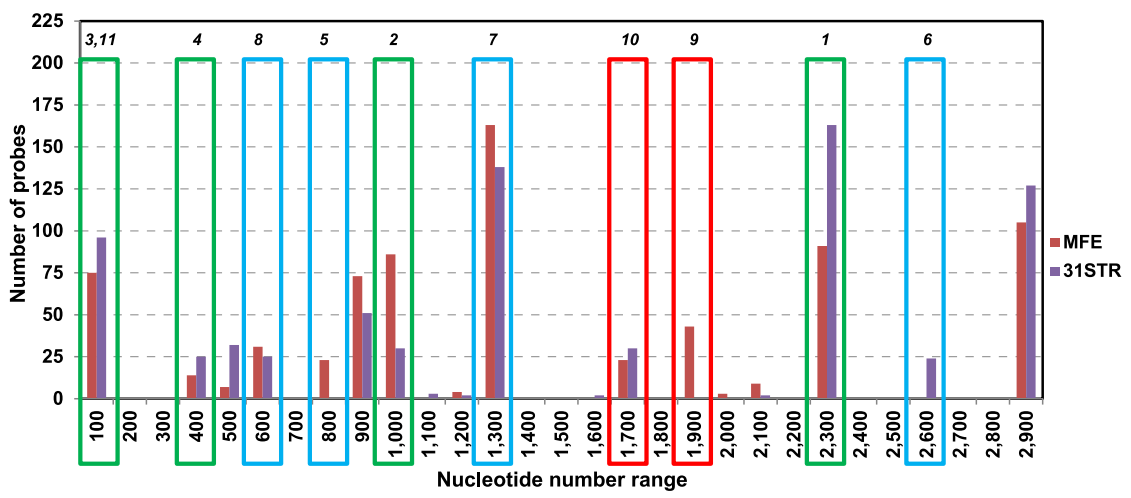

FIGURE 4. Comparison of target regions selected by the PinMol program for designing osk mRNA molecular beacons (bars) with experimental results (boxes). The distribution of the probes (bars) designed using a target ss-count file generated with mfold: MFE-red, 31STR -purple, for probe lengths between 14 and $28 \mathrm{nt}$. Red boxes show molecular beacons that performed poorly experimentally; blue boxes show probes that have a moderate efficiency to hybridize to their target region, and green boxes highlight probes that hybridize to their target region with the highest efficiency. The numbers on top of the boxes are the in vitro experimental (RNA hybridization) rankings of each probe, with one being the most efficient probe.

\section{Optimizing the molecular beacon design for live cell imaging}

Ideally, when designing molecular beacons for live cell imaging experiments, the in vivo target mRNA secondary structure should be taken into consideration when selecting the probe sequence. While in vivo mapping has been proven useful in determining mRNA secondary structure in live cells and tissues (Mathews et al. 2004; Rouskin et al. 2014), due to the scarcity of such data, molecular beacon design relies on mRNA secondary structures predicted via free energy minimization approaches. Moreover, it was reported that active unfolding of mRNA structures occurs in vivo (Rouskin et al. 2014). For osk mRNA 

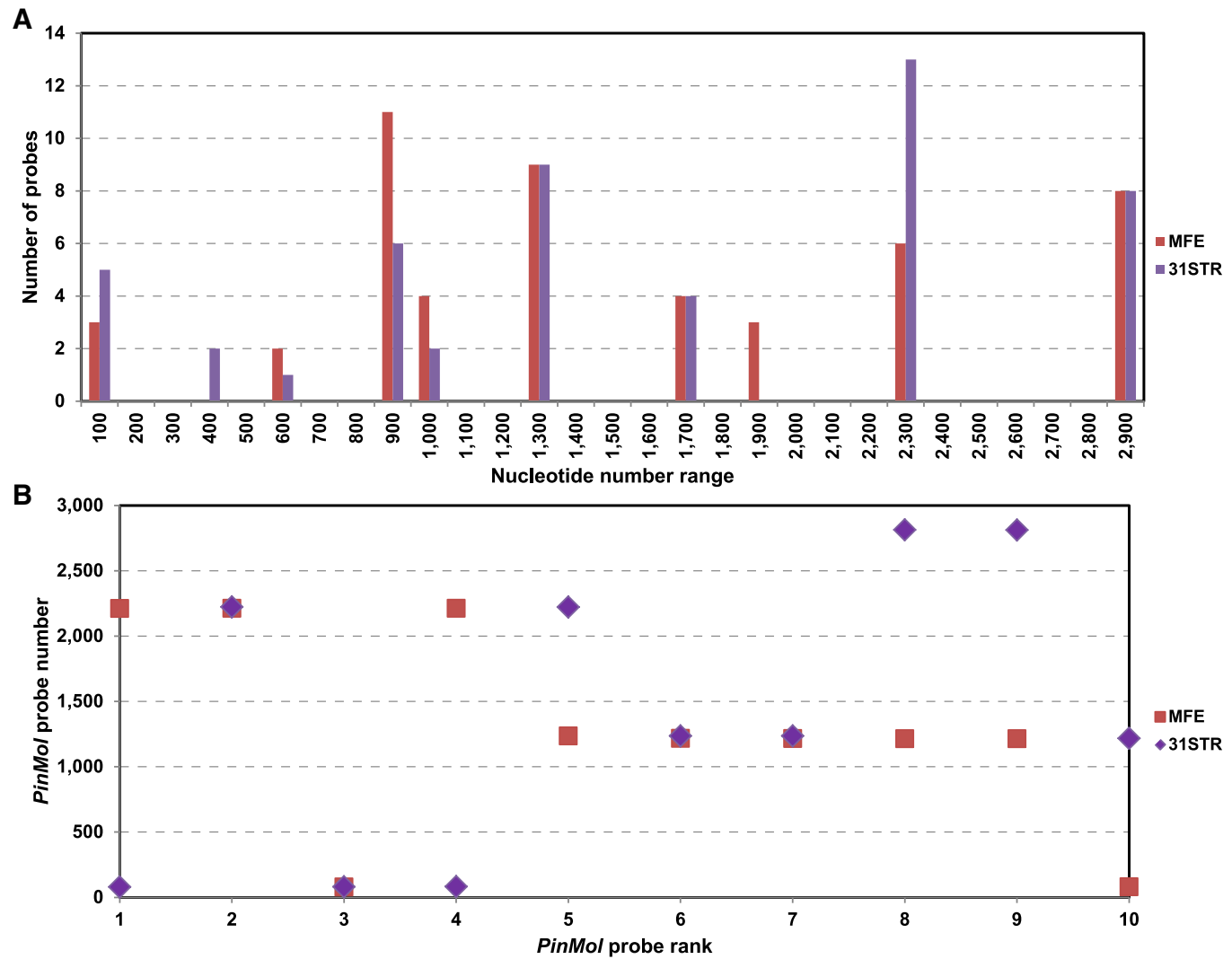

FIGURE 5. Molecular beacons with $24 \mathrm{nt}$ probe length designed with the PinMol program for osk mRNA. (A) The distribution of the probes (bars) designed using a target ss-count file generated with mfold: MFE—red, 31STR—purple. (B) Top 10 probes designed with PinMol for the input files indicated in $\mathrm{A}$.

probe design, when including structural information from 31STR versus MFE, PinMol allowed identification of additional regions that are viable targets in in vivo experiments, by taking into consideration the mRNA's folding flexibility.

Three popular RNA folding programs, mfold, RNAstructure, and Vienna RNAfold, use different thermodynamic parameter sets and therefore, do not predict identical structures, but they are accepted to have similar prediction accuracies. As a consequence, probe ranking using PinMol is also expected to differ when using input files generated with each of these programs. However, regardless of the program used to generate the PinMol input file, valid molecular beacon candidates will be obtained. Although we recommend using the ss-count file generated by mfold, analyzing and comparing target structures predicted by both mfold and RNAstructure may reveal regions that are consistently predicted to be more accessible, which will maximize the chances of designing highly efficient molecular beacons for live cell imaging experiments. RNAstructure does not yield an ss-count output file, but we converted the ".ct" output file into an ss-count formatted file using a Python script, which is available upon request.
To test the ranking accuracy of PinMol selected probes when using mfold-generated input files, we selected molecular beacon osk1236 with a probe length of $24 \mathrm{nt}$ (for which the $5^{\prime}$ end of the stem coincidently includes three additional nucleotides matching the target) to compare it to osk82 and osk2216 (Fig. 6A). We chose the probe length of $24 \mathrm{nt}$ in order to be consistent with the probe length for both, osk82 and osk2216. Probe osk1236 was the highest ranking probe that is complementary to a target region that is distinct from the regions targeted by the experimental probes osk82 and osk2216 (Supplemental Table S1). osk1236 was ranked 6th by PinMol, behind five probes $(80,2223,81,82$, and 2222) that are all positioned very closely to the target regions where the two experimental probes hybridize. The osk2216 molecular beacon had the best performance, while osk82 placed third in in vitro hybridization experiments (Table 2; Bratu et al. 2003), and osk2216 was also efficient in detecting endogenous osk mRNA in in vivo experiments (Catrina et al. 2012). When comparing their $f_{\text {ss-count }}$ values, osk1236 was predicted to outrank osk2216 for both mfold-generated input files (MFE and 31STR, Table 3). However, osk82 outranked osk1236 when using the 31STR ss-count file. 


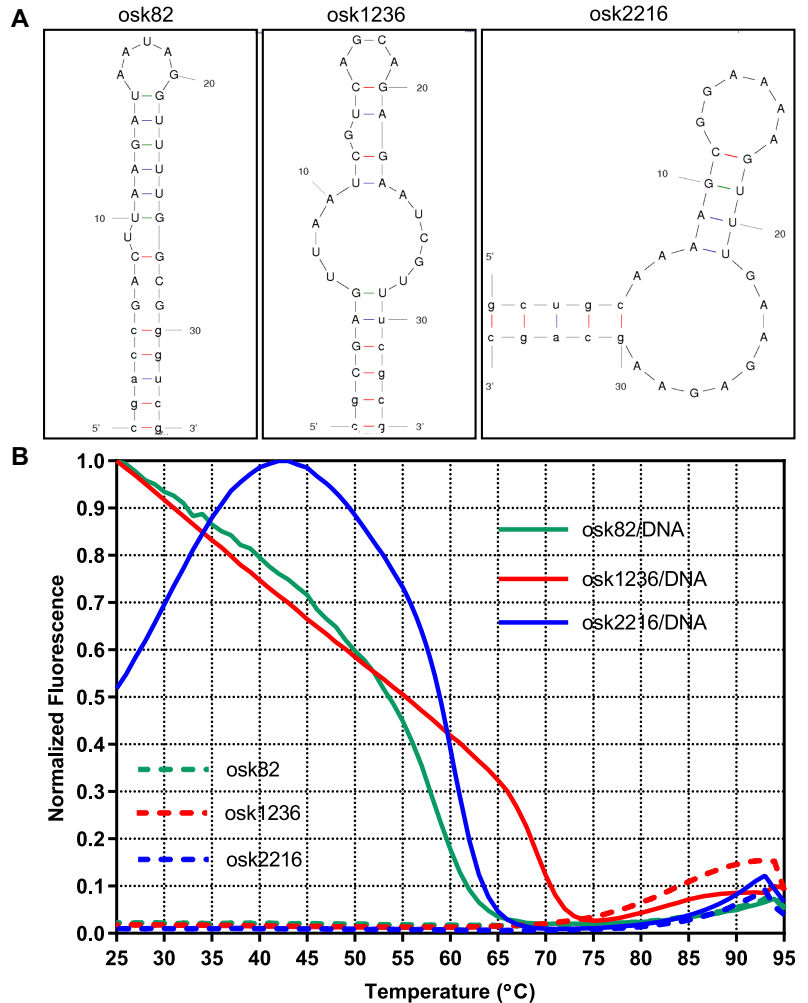

FIGURE 6. Comparison of the thermal denaturation profiles of molecular beacons osk82, osk1236, and osk2216. (A) The mfold predicted MFE structure for each molecular beacon. (B) Thermal denaturation curves for molecular beacon alone (dashed lines), or molecular beacon and DNA oligonucleotide target (solid lines): osk82 (green), osk1236 (red), and osk2216 (blue).

We synthesized and purified osk1236 (Materials and Methods) and when comparing the thermal denaturation profiles for these three molecular beacons, we found that osk1236 had a slightly lower Tm than osk2216 and osk82, consistent with predicted mfold results $\left(82^{\circ} \mathrm{C}\right.$ vs. $88^{\circ} \mathrm{C}$ and $86^{\circ} \mathrm{C}$, Table 4 ; Fig. 6B). For thermal denaturation profiles of molecular beacon in the presence of DNA oligonucleotide target, there was a similar trend with osk1236/ DNA having a lower $\mathrm{Tm}$ than osk2216/DNA, but not osk82/DNA $\left(55^{\circ} \mathrm{C}\right.$ vs. $59^{\circ} \mathrm{C}$ and $53^{\circ} \mathrm{C}$, Table 4 ; Fig. $\left.6 \mathrm{~B}\right)$, although osk1236/DNA contains a higher percentage of G/C base pairs when compared to osk2216/DNA (GC\%: osk82, 37.5 , osk1236, 41.7, and osk2216, 33.3). The thermal dena- turation profile of osk2216 with its DNA oligonucleotide complement is complicated by the combination of the probes' low percentage of GC nucleotides and moderately favorable $\Delta \mathrm{G}_{\text {bimol }}(-17.4 \mathrm{kcal} / \mathrm{mol}$ for the osk 2216 molecular beacon sequence and $-4.4 \mathrm{kcal} / \mathrm{mol}$ for the probe sequence). When using a DNA oligonucleotide target, the intermolecular interaction of osk2216 molecular beacon is favored over the formation of osk2216/DNA hybrid, and as a result the molecular beacon falls off the target DNA at temperatures lower than $43^{\circ} \mathrm{C}$, which led to a sharp decrease in fluorescence signal (Fig. 6B). Because RNA/ RNA hybrids are energetically more stable than RNA/ DNA hybrids, this behavior is less likely to occur when the molecular beacon hybridizes with its intended mRNA target region. We found that the hybridization half-life of osk1236 with the corresponding DNA oligonucleotide target is slightly longer than that of osk2216 and osk82 molecular beacon (12.6 vs. 1.2 and $2.6 \mathrm{sec}$ ) (Table 4; Fig. 7A). However, for hybridization reactions with full-length, in vitro transcribed osk RNA, we observed that osk2216/RNA $(49.0 \mathrm{sec})$ and osk82/RNA $(47.8 \mathrm{sec})$ hybrids had a much shorter half-life than the osk1236/RNA hybrid $(480.4 \mathrm{sec})$ (Table 4; Fig. 7B).

We analyzed and quantified the in vivo performance of each molecular beacon, osk82, osk1236, and osk2216. Quantification of molecular beacon in vivo target detection efficiency is not trivial due to several variables that need to be controlled that are expected to result in a wide variation in the final results, which we observed for osk1236 (Table 5). We took into account some of these variables:

1. Molecular beacon quantity delivered; to control for how much molecular beacon is injected in each egg chamber, we added to the molecular beacon injection solution DNA oligonucleotides labeled with a spectrally different fluorophore (TMR), which we designed for use in RNA single molecule fluorescence in situ hybridization (smFISH) specific to gurken mRNA (Bayer et al. 2015).

2. Proximity of the injection site to the cellular location visualized; as much as possible the injections were performed within a nurse cell proximal to the oocyte.

3. Stage of the development of the microinjected egg chamber; we selected stage 8 to late stage 9 egg

TABLE 3. Comparison of parameters for the probe sequence of three molecular beacons targeting different regions of osk mRNA

\begin{tabular}{|c|c|c|c|c|c|c|}
\hline \multirow[b]{2}{*}{ Parameters } & \multicolumn{3}{|c|}{ MFE } & \multicolumn{3}{|c|}{ 31STR } \\
\hline & osk82 & osk1236 & osk2216 & osk82 & osk1236 & osk2216 \\
\hline$f_{\text {ss-count }}$ & 0.750 & 0.792 & 0.667 & 0.763 & 0.743 & 0.694 \\
\hline Rank & 20 & 5 & 52 & 4 & 6 & 25 \\
\hline
\end{tabular}

(MFE) Minimum free energy structure. (31STR) MFE and 30 suboptimal structures. 
TABLE 4. In vitro hybridization results for osk82, osk1236, and osk2216 molecular beacons

\begin{tabular}{llcc}
\hline Parameters & osk82 & osk1236 & osk2216 \\
\hline Predicted $\Delta \mathrm{G}$ folding $(\mathrm{kcal} / \mathrm{mol})^{\mathrm{a}}$ & -7.9 & -6.6 & -6.7 \\
$\mathrm{Tm} \mathrm{MB}\left({ }^{\circ} \mathrm{C}\right)$ & 86 & 82 & 88 \\
$\mathrm{Tm} \mathrm{MB} / \mathrm{DNA}\left({ }^{\circ} \mathrm{C}\right)$ & 53 & 12.6 & 59 \\
Half-life MB/DNA at $25^{\circ} \mathrm{C}(\mathrm{sec})$ & 2.6 & 480.4 & 1.2 \\
Half-life MB/RNA at $25^{\circ} \mathrm{C}(\mathrm{sec})$ & 47.8 & $80.9 \pm 3.3$ & 49.0 \\
Signal-to-background ratio at $25^{\circ} \mathrm{C}(\mathrm{MB} / \mathrm{DNA})^{\mathrm{b}}$ & $161.5 \pm 9.5$ & & $119.8 \pm 2.7$ \\
\hline (MB) Molecular beacon. & & & \\
${ }^{\mathrm{M} M F E}$ mfold. & & &
\end{tabular}

chambers for microinjections, in which the osk mRNA target is mainly localized at the posterior end of the oocyte; to account for the variations in the size of the oocyte, we used a correction factor to adjust the calculated relative detection efficiency of a molecular beacon in larger versus smaller oocytes (Equation 2).

4. Depth of image acquisition to collect the optical slice with maximum fluorescence signal; we acquired 14 optical slices $(0.3 \mu \mathrm{m}$ each) and chose the $\mathrm{XY}$-plane with maximum signal in the selected region of interest (ROI).

5. Timing of the initiation of acquisition protocol; we initiated acquisition immediately after acquiring the Z-stack for the TMR channel injection control (also see Materials and Methods); to control for any delay in the start of data acquisition, we quantified the change in the Cy5 signal intensity within the posterior $\mathrm{ROI}$ from time point $t 1$ ( $0 \mathrm{~min})$ to $t 3(4 \mathrm{~min})$.
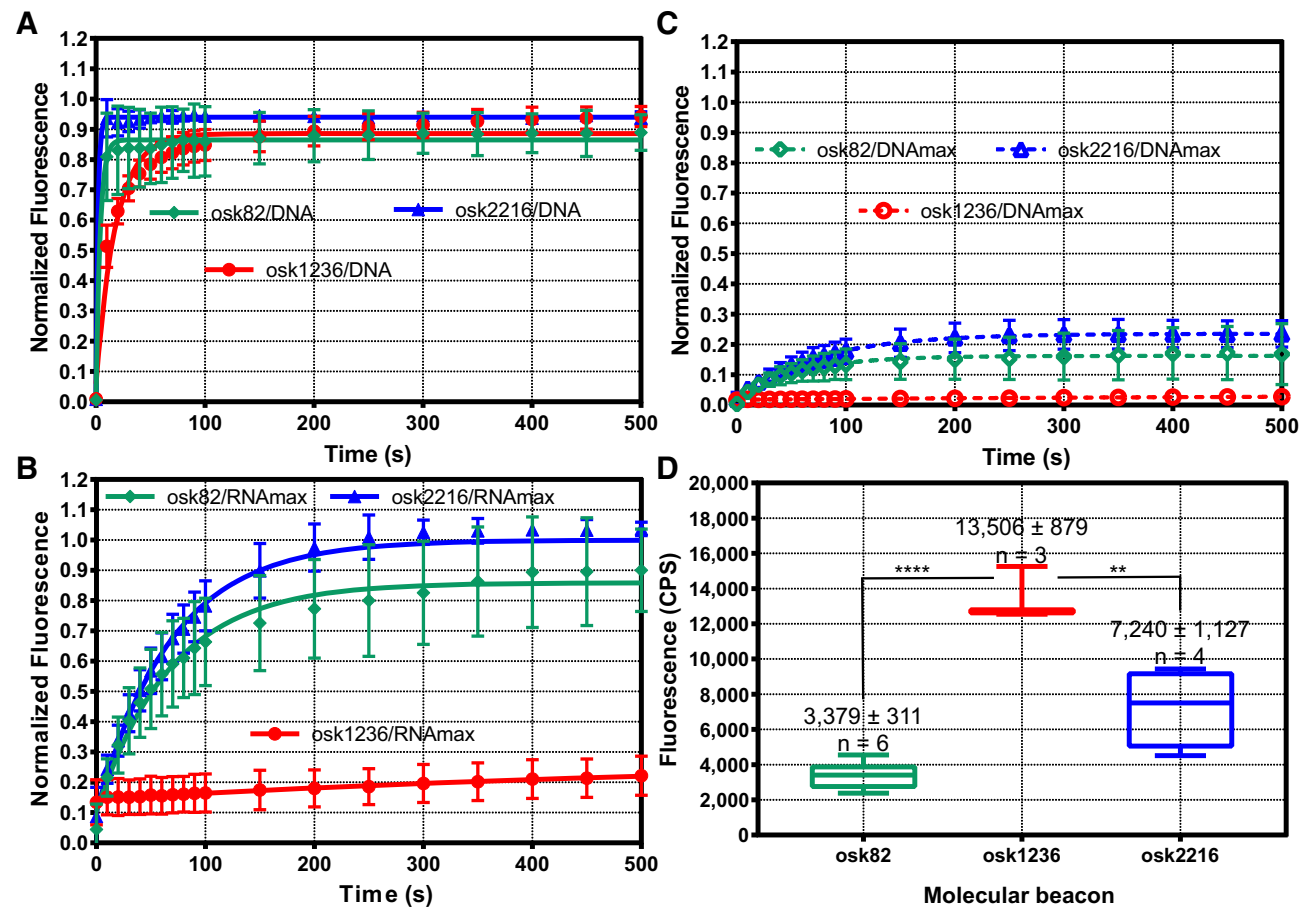

FIGURE 7. Comparison of in vitro molecular beacon/target hybridization profiles of osk82, osk1236, and osk2216. Hybridization curves for each molecular beacon with the corresponding complementary (A) DNA oligonucleotide target (MB/DNA) (osk82-green solid line, osk1236-red solid line, osk2216 — blue solid line, filled symbols), or (B) and (C) with full-length, in vitro transcribed osk RNA (osk82—green, osk1236—red, osk2216-blue). In B are shown the RNA hybridization curves normalized using the fluorescence signal from first plateau of the RNA hybridization reaction (MB/RNAmax, solid lines, filled symbols). In C, for comparison, the fluorescence signal (CPS) for the RNA hybridization curves was normalized to the maximum fluorescence signal obtained upon adding DNA target after the RNA hybridization reaction reached the first plateau (dashed lines, open symbols). (D) Average maximum fluorescence signal (CPS) from in vitro RNA hybridization experiments for each molecular beacon (floating bars: osk82 [green], osk1236 [red], osk2216 [blue], mean \pm SEM unpaired t-test: $\left.\left(^{* * * *}\right) P<0.0001,\left(^{* *}\right) P<0.0092\right)$. MB, molecular beacon (i.e., osk82, osk1236, or osk2216). 
TABLE 5. Relative detection efficiencies, quantification, and statistical analysis of molecular beacon in vivo imaging results

\begin{tabular}{lccc}
\hline Molecular beacon & Efficiency \pm SEM & $P^{a}$ & No. of injections \\
\hline osk82 & $0.130 \pm 0.015$ & $* * * /<0.0004$ & 8 \\
osk1236 & $0.844 \pm 0.163$ & $\mathrm{n} / \mathrm{a}$ & 7 \\
osk2216 & $0.162 \pm 0.021$ & $* * /<0.0013$ & 7 \\
\hline aUnpaired t-test for comparing in vivo detection efficiency of osk1236 with osk82 and osk2216.
\end{tabular}

Despite osk1236's less efficient in vitro performance with target RNA when compared to that of osk2216 and osk82, osk1236 presented a superior fluorescence signal in in vivo experiments (Fig. 8; Table 5: 0.84 vs. 0.16 and 0.13 relative detection efficiency; Materials and Methods; Supplemental Movies S1-S4). Although we used the same fluorophore for all molecular beacons (Cy5), the two previously synthesized molecular beacons (osk82 and osk2216) had an aged fluorophore, which could result in a decreased fluorescence signal as compared to osk1236. To evaluate if this could have impacted our quantitative analysis, we determined how the performance of the three osk molecular beacons compared in in vitro hybridization experiments with osk RNA target. We determined the average maximum fluorescence for each molecular beacon (Fig. 7D), and calculated the normalized fluorescence after 4 min, which is the time point when the in vivo fluorescence signal was quantified. This value is the fraction of the fluorescence signal at 4 min after the RNA target was added from the possible maximum in the presence of RNA target for each molecular beacon (Fig. 7B; osk82: 0.8, osk1236: 0.19 , and osk2216: 1). After 4 min, the maximum raw fluorescence signal (CPS) was obtained for osk2216, followed by osk 82 and osk 1236 (37\% and 35\% of osk2216 CPS, respectively). Although based on the in vitro RNA hybridization results of the three osk molecular beacons, we would expect osk2216 to yield the highest raw fluorescence signal in vivo, we found that osk1236 outperformed both, osk2216 and osk82. This was evident when comparing in vivo imaging results for stage 8 egg chambers microinjected with similar amounts of the corresponding molecular beacon solution, which were acquired and processed using the same parameters, and where a bright fluorescence signal is observed only for osk1236 (Supplemental Movie S1).

It is possible that the regions targeted by osk 82 and osk2216 are not as easily accessible in vivo due to binding of protein factors to osk mRNA, which would lead to a decreased detection efficiency as compared to in vitro results. It is also possible that the RNA region targeted by osk1236 is more rigid in solution than in vivo, which leads to a longer osk1236/RNA hybridization half-life. We found that when using mfold-generated input files, osk2216 is highly ranked by PinMol (25th probe) when also taking into consideration target suboptimal structures, but it is not selected in the top 50 probes when using MFE as PinMol input file (52nd probe).

Taken together, these results suggest that when incorporating information from predicted target RNA suboptimal secondary structures in addition to the MFE structure, the design of molecular beacon probes for in vivo experiments improves (Table 2).

\section{Selecting the probe length}

PinMol allows the design of up to 50 probes with lengths in a range of 18-26 nt. How does one decide what probe length will work best for a particular target or target region? This should not pose a problem when the targeted region is thousands of nucleotides long, but when using a short target, probe selection will be constrained by additional factors. The number of probes meeting all criteria ( $\Delta$ G-probes) significantly decreased for probe lengths larger than $22 \mathrm{nt}$ (Fig. 3B), indicating that this number may be

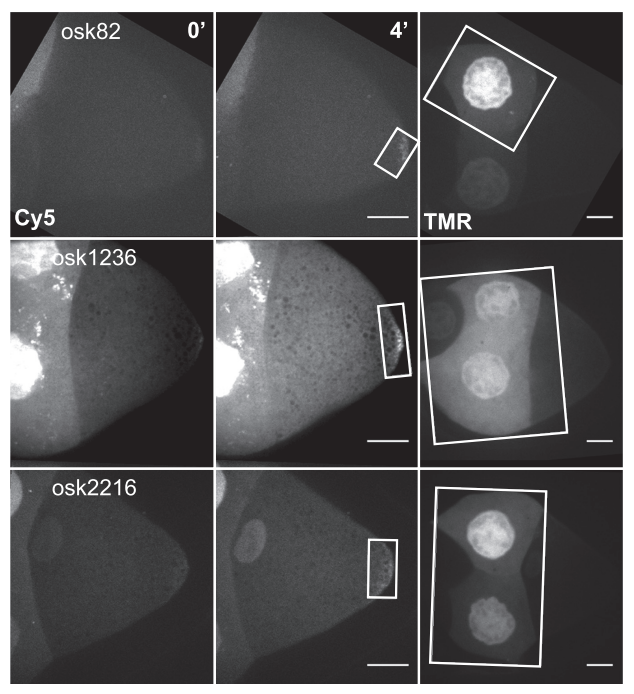

FIGURE 8. Visualization and quantification of the detection efficiency of endogenous osk mRNA in live cell imaging. Fluorescence signal generated by molecular beacon osk82, osk1236, or osk2216 microinjected in late stage 9 wild-type fruit fly egg chambers, at the indicated time points. White rectangles indicate the ROIs used for quantification analysis of fluorescence signal generated by each molecular beacon (Cy5), and includes a control for the amount of each injected molecular beacon (TMR). Scale bar is $20 \mu \mathrm{m}$. 
an optimal length. However, if $\mathrm{A} / \mathrm{T}$ - or $\mathrm{G} / \mathrm{C}$-rich regions are targeted, in order to meet all requirements as implemented in PinMol, a longer or shorter probe length should be selected, respectively.

Ultimately, the main consideration for designing the probe sequence was target accessibility, which was taken into account by sorting all possible probes in descending order of the $f_{\text {ss-count. }}$. PinMol does not limit the $f_{\text {ss-count }}$ to a lower bound (e.g., consider only probes that target a region with a $f_{\text {ss-count }}>0.5$ ) because it limits the total number of selected probes to 50 , or the maximum number of possible probes, whichever is smaller. Taking into consideration our previous experimental results, optimization of other parameters (e.g., probe length) is also required for the selection of the most efficient probe(s) for live cell imaging. Therefore, it is important to know which factors are most useful in predicting highly efficient probes. We first analyzed our previous experimental data, and we found that only two molecular beacons showed high efficiency in detecting the target region in in vitro experiments, while the remaining nine molecular beacons were all under the $50 \%$ hybridization efficiency as compared to osk2216, our best experimental probe (Fig. 9; Bratu et al. 2003). When we compared the top two probes, the $f_{\text {ss-count }}$ appeared to be sufficient to predict their rank (0.694 vs. $0.616,31 \mathrm{STR}$ ) (Table 2). When we compared the first two probes with the third (osk82), although they have similar probe lengths, osk82 had the highest $f_{\text {ss-count }}(0.76)$ when considering $30 \mathrm{SO}$ structures in addition to the MFE structure, but it also had a more stable bimolecular folding

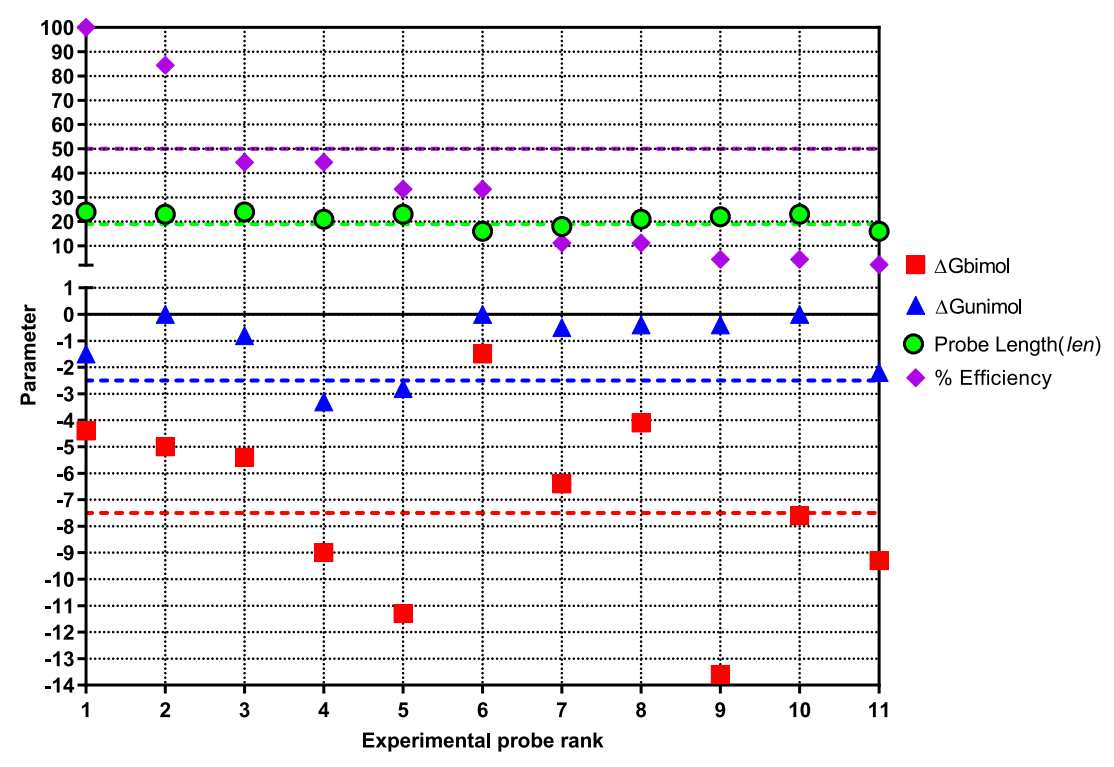

FIGURE 9. Parameters used by PinMol for probe selection. oligoscreen predicted values for the probe sequence of experimental molecular beacons previously reported for osk mRNA (Table 2; Bratu et al. 2003). Colored dashed lines highlight the limits on probe selection as imposed by PinMol for design of molecular beacons suitable for live cell imaging. All probes are shorter than the upper limit of probe length, thus only the lower limit is presented.
(Table 2; Fig. 9). This suggests that the $\Delta \mathrm{G}_{\text {bimol }}$ may be another important determinant of probe efficiency for probe lengths in the range of 21-24 nt. The probes ranked experimentally as 4th, 5th, 9th, 10th, and 11 th were not selected by $P$ inMol because they have a $\Delta \mathrm{G}_{\text {bimol }}<-7.5 \mathrm{kcal} / \mathrm{mol}$, and the first two probes also have a $\Delta \mathrm{G}_{\text {unimol }}<-2.5 \mathrm{kcal} /$ mol. Therefore, these in vitro data support our assessment that probes with favorable uni- and/or bimolecular folding will perform poorly (Fig. 9). Of the remaining probes (Table 2), osk2585 ranked 6th and osk1220 ranked 7th showed a decrease in performance that was directly related to their $f_{\text {ss-count, }}$ with osk2585 (0.76) having a larger $f_{\text {ss-count }}$ than osk1220 (0.68), and also presented a more stable bimolecular folding energy (Table 2; Fig. 9). However, osk2585 showed a slightly lower efficiency than two probes that were filtered out by PinMol (osk358 and osk720, experimentally ranked 4 th and 5 th, Table 2 ). It is possible that the shorter probe length ( 16 vs. 21 and $23 \mathrm{nt}$ ) strongly impacted the performance of the former, and thus we chose $18 \mathrm{nt}$ as the minimum length of probe sequence. Finally, the molecular beacon experimentally ranked 8th (osk544) presented the second lowest $f_{\text {ss-count }}$ (0.512), which explains its poor performance in in vitro hybridization experiments with full-length osk mRNA (Table 2).

Inter- or bimolecular folding is correlated with the molecular beacon's concentration, and it may negatively impact purification of the probe when using concentrated stock solutions. However, its impact on in vivo experiments is expected to be somewhat reduced since lower concentrations are used. Therefore, instead of setting a stringent cut-off for $\Delta \mathrm{G}_{\text {bimol, }}$, we chose a moderately low value of $-7.5 \mathrm{kcal} / \mathrm{mol}$. For comparison, the $\Delta G_{\text {bimol }}$ folding of the osk2216 probe region with a fully complementary RNA oligonucleotide was predicted using the oligoscreen subroutine to be $-35.3 \mathrm{kcal} / \mathrm{mol}$ $\left(\Delta \mathrm{G}_{\text {duplex }}\right.$ in Fig. 2: \#5, "probes sortedby5.csv" PinMol output file).

We determined the number of molecular beacons designed by PinMol for each probe length when using the 31STR input file generated with mfold. For all probe lengths, the average final number of molecular beacons was 27 .

\section{DISCUSSION}

PinMol facilitates the design of molecular beacons for live cell imaging experiments. We have tested the performance of PinMol by comparing the ranking of PinMol designed molecular beacons with experimental 
results for manually selected molecular beacons specific for osk mRNA, a well-studied fruit fly maternal mRNA. Based on our analysis, we propose the following guidelines when designing molecular beacons for live cell imaging:

1. Use the mfold-generated ss-count, and consider target suboptimal secondary structures with a change in folding free energy within 5\%-10\% of the MFE.

2. Avoid probes complementary to the ends of the $5^{\prime}-$ UTR and $3^{\prime}-U T R$ of the mRNA target: These regions are usually targeted by cellular factors and/or form long distance base pairs; this is supported by the observed low in vivo efficiency for osk82, despite showing a strong performance in in vitro experiments.

3. Select the length of the probe sequence between 18 and $26 \mathrm{nt}$ : We propose an optimum length of $22 \mathrm{nt}$, but this is ultimately dependent on the base composition of the targeted region, which will require longer probes for A/U-rich and shorter probes for G/C-rich sequences.

4. If possible, avoid a G nucleotide at the $5^{\prime}$ end of the molecular beacon: It can quench the fluorescence of certain fluorophores (Marras et al. 2002; Nazarenko et al. 2002).

5. Perform BLAST alignments: Carefully evaluate any cross homology by taking into consideration if the target mRNA shows tissue-specific expression.

\section{MATERIALS AND METHODS}

\section{PinMol software}

The PinMol software was written using Python 3.5.2, for use on Mac and Windows platforms, and packaged for easy download from GitHub (https://github.com/icatrina/PinMol_Mac and https ://github.com/icatrina/PinMol_Win). PinMol requires Python 3, Pandas, Biopython, and text-interface RNAstructure (requires free registration, and it is available for download from the Mathews laboratory webpage at the University of Rochester: http ://rna.urmc.rochester.edu/RNAstructureDownload.html).

PinMolv1.0 was tagged and released using Git.

Detailed tutorials for the Mac version with or without BLAST analysis, the input files used to design osk- and nanos-specific molecular beacons with PinMol, as well as the output files from designing osk-molecular beacons for 24 nt-long probes using mfold-generated 31STR input file, are available on our laboratory's homepage at https://bratulab.wordpress.com/software/.

\section{Secondary structure prediction}

The MFE and suboptimal RNA target secondary structures were predicted using the RNA form of mfold with RNA3.5 parameters. The ss-count files containing information on MFE and selected suboptimal structures were saved as simple text files. Since there is no ss-count file for the MFE structure alone, we converted the corresponding ".ct" files as follows: We copied the first and sec- ond columns and replaced the length of the folded RNA target at the top of the first column with "1," and added information from column five by replacing the " 0 " representing a single-stranded nucleotide with "1," and also replacing a nucleotide number representing a binding partner with " 0. "

\section{Molecular beacon design, synthesis, and purification}

The molecular beacons osk2216, Cy5-C6-gcugcAAAAGCGGAA AAGUUUGAAGAGAAgcagc-BHQ-2, and osk82 Cy5-C6-cgacc GACUUAAGAUAAUAGGUUUUGGCGggucg-BHQ-2 are the previously described and characterized osk76 and osk2209 molecular beacons (Bratu et al. 2003; Mhlanga et al. 2009). The $2^{\prime} \mathrm{OMe}$ oligonucleotide labeled with a 5' C6-amino linker and a 3' BHQ-2 corresponding to osk1236 sequence, NH2-C6-cgCGA GUUAAUCGUCAGCAGAGAAUCGUUucgcg-BHQ-2, was synthesized on the 100 nmole scale by Integrated DNA Technologies. The oligonucleotide was labeled with amino-reactive Cy5 and purified using HPLC as previously described (Bratu et al. 2011). CAPS show the single-stranded probe sequence, italics show the stem region, and small letters represent the newly added nucleotides as part of the stem region. The sequences for the complementary DNA oligonucleotides used for in vitro molecular beacon-target hybridization reactions are as follows: osk82.c CCGCCAAAACCTATTATCTTAAGTCC, osk1236.c CCTACAAC GATTCTCTGCTGACGATTAACTCGGAT, and osk2216.c AAAAC TTCTCTTCAAACTTTTTCCGCTTTTCCCAA.

\section{In vitro thermal denaturation and hybridization experiments}

Thermal denaturation reactions were run with $300 \mathrm{ng}(\sim 190 \mathrm{nM})$ of molecular beacon alone or with $3 \mu \mathrm{g}(\sim 2.3 \mu \mathrm{M})$ complementary DNA oligonucleotide target. For molecular beacon thermal denaturation, the reaction was cooled down from $95^{\circ} \mathrm{C}$ to $25^{\circ} \mathrm{C}$, at a rate of $1^{\circ} \mathrm{C} / 30 \mathrm{sec}$. For thermal denaturation experiments in the presence of complementary DNA oligonucleotide target, $3 \mu \mathrm{L}$ of DNA oligonucleotide target were added to $120 \mu \mathrm{L}$ containing $300 \mathrm{ng}$ molecular beacon in hybridization buffer $(50 \mathrm{mM}$ Tris- $\mathrm{HCl}$, $\mathrm{pH} 7.5,1.5 \mathrm{mM} \mathrm{MgCl}_{2}$ and $100 \mathrm{mM} \mathrm{NaCl}$ ). Experiments were performed in a FluoroMax-4 photon counting spectrofluorometer (Horiba-Jobin Yvon), and for each temperature, three time points were acquired every $15 \mathrm{sec}$, after $0.2 \mathrm{~min}$ temperature equilibration with an integration occurring over $0.5 \mathrm{sec}$. The average of the three readings for each temperature was graphed using GraphPad Prism. The absolute fluorescence intensity (CPS) was normalized for each molecular beacon to the maximum CPS signal obtained for the thermal denaturation of molecular beacon with DNA oligonucleotide target. The excitation and emission slit widths were each $3 \mathrm{~nm}$ and $2 \mathrm{~nm}$, respectively, with excitation/emission wavelengths of 649/670 nm.

For in vitro hybridization reactions using complementary DNA oligonucleotide targets, $100 \mathrm{ng}(\sim 130 \mathrm{nM} ; 60 \mu \mathrm{L}$ reaction volume) of the molecular beacon was incubated with $500 \mathrm{ng}(\sim 780 \mathrm{nM})$ of the corresponding complementary DNA oligonucleotide target. The fluorescence signal was acquired using a FluoroMax-4 photon counting spectrofluorometer (Horiba-Jobin Yvon), and was monitored at $25^{\circ} \mathrm{C}$, first with just the hybridization buffer, then with the molecular beacon, and lastly with the corresponding complementary DNA oligonucleotide target. It was recorded 
every $2 \mathrm{sec}$, with an integration occurring over $0.5 \mathrm{sec}$. The excitation and emission slit widths were each $2 \mathrm{~nm}$, with excitation/ emission wavelengths of $649 / 670 \mathrm{~nm}$. The signal-to-background ratio was calculated by dividing the maximum fluorescence signal (CPS) by the difference between the fluorescence signal of the molecular beacon alone and the buffer background.

In vitro transcription of full-length osk RNA, and hybridization reactions with molecular beacons and osk RNA were performed as previously described (Catrina et al. 2012). Briefly, the hybridization reaction was performed in hybridization buffer $(60 \mu \mathrm{L}$ total volume: $80 \mathrm{nM}$ molecular beacon was incubated with $60 \mathrm{nM}$ folded osk RNA), and the change in fluorescence signal was recorded at $25^{\circ} \mathrm{C}$ every $10 \mathrm{sec}$, with $2 \mathrm{sec}$ integration time and using $3 \mathrm{~nm}$ and $2 \mathrm{~nm}$ excitation $(649 \mathrm{~nm})$ and emission $(670 \mathrm{~nm})$ slit widths, respectively. The RNA was folded in hybridization buffer two ways: (i) incubation at $95^{\circ} \mathrm{C}$ for $10 \mathrm{sec}$ and immediately placed on ice, and (ii) incubation at $70^{\circ} \mathrm{C}$ for $10 \mathrm{~min}$, followed by slow cooling over $30 \mathrm{~min}$ to $30^{\circ} \mathrm{C}$ and placed on ice until ready to use. The sense strand of full-length osk mRNA was in vitro transcribed using a T7MegaScript Ambion Kit (ThermoFisherAmbion), following the manufacturer's instructions using a PCR fragment amplified from a plasmid containing osk cDNA inserted into a pBluescript KS+ vector (kind gift from R. Lehmann, NYU Medical School). After the RNA hybridization reaction reached a first plateau (for osk82 and osk2216) we added $3 \mu \mathrm{g}$ of complementary DNA oligonucleotide and continued the acquisition until a maximum plateau was reached. For DNA hybridization analysis, the raw fluorescence signal (CPS) was normalized the maximum fluorescence signal, and data were fitted using nonlinear regression for binding kinetics of one phase association (GraphPad Prism). For RNA hybridization analysis, the raw fluorescence signal (CPS) was normalized the maximum fluorescence signal with target osk RNA using the first plateau obtained from fitting the hybridization data as described above, or using the maximum fluorescence signal when adding complementary DNA oligonucleotide to the RNA hybridization reaction after it reached the first plateau. The graphs show representative curve profiles (Fig. 6) or nonlinear fit using GraphPad Prism (Fig. 7) from three or more independent thermal denaturation, or hybridization experiments, respectively. The average maximum fluorescence signal (CPS) from in vitro RNA hybridization data was calculated with GraphPad Prism using the values fitted for the first plateau, as described above.

\section{Live cell imaging in D. melanogaster oocytes and image quantification}

Newly hatched Oregon R-P2 (Bloomington stock \#2376) female flies were fed fresh yeast paste for 2-4 $d$. Whole ovaries were dissected in Halocarbon Oil 700 (Sigma-Aldrich) directly on a glass coverslip and then teased apart into individual egg chambers. After mounting the glass coverslip onto the stage of the spinning disc confocal microscope, a solution containing $300 \mathrm{ng} / \mu \mathrm{L}(\sim 23$ $\mu \mathrm{M})$ molecular beacon and $30 \mathrm{ng} / \mu \mathrm{L}$ TMR-labeled DNA probes specific to gurken mRNA designed for smFISH ( 22 nt in length) in hybridization buffer was microinjected into the nurse cell most proximal to the oocyte. As control for the molecular beacon quantity injected, we acquired an optical stack of 41 slices $(1 \mu \mathrm{m}$ each) for the TMR channel only and with a $40 \times$ objective (oil, NA
=1.25). Image acquisition of $X Y Z C t(X, Y, Z$, channel, time) stacks was performed with a $63 \times$ objective (oil, $N A=1.4$ ) for TMR and Cy 5 channels, 14 optical slices $(0.3 \mu \mathrm{m}$ each), every 2 min for up to $30 \mathrm{~min}$, on a Leica DMI-4000B inverted microscope (Leica Microsystems Inc.) mounted on a TMC isolation platform (Technical Manufacturing Corporation), and equipped with a Yokogawa CSU 10 spinning disk head and Hamamatsu C910013 ImagEM EMCCD camera (Perkin-Elmer), $561 \mathrm{~nm}$ and $635 \mathrm{~nm}$ diode lasers (Spectra Services Inc.), and an Eppendorf Patchman-Femtojet microinjector (Eppendorf Inc.). The images were acquired using Volocity 6.3.0 acquisition software (Perkin-Elmer) and then processed with Fiji/lmageJ software (NIH) (Schindelin et al. 2012). For Supplemental Movie S1, after maximum intensity $\mathrm{XY}$-projection of Z-stack, the brightness and contrast were adjusted for all 16-bit acquisition files using values optimal for osk1236, which presented the brightest in vivo fluorescence signal of all three osk molecular beacons. For Supplemental Movies S2-S4, after maximum intensity $\mathrm{XY}$-projection of Z-stack, the resulting time series were brightness and contrast adjusted using optimal values for each molecular beacon.

In order to compare the detection efficiency of the three molecular beacons, we quantified the Cy5 fluorescence signal accumulated at the posterior of the oocyte at the initiation of acquisition $(t 1=0 \mathrm{~min})$ and after $4 \mathrm{~min}(t 3=4 \mathrm{~min})$, for three developmental stages (stage 8, 9, and late 9). We used identical acquisition parameters (laser power, and exposure time, gain and sensitivity for high-quality camera acquisition mode) for all microinjections, and found that when using parameters necessary for a detectable fluorescence signal for osk82 and osk2216 molecular beacons, time points after 4 min presented a saturated fluorescence signal for osk1236. We cropped the posterior ROI for both time points and measured the ROI area and the raw intensity density (RID) for each of the 14 optical slices at each of the two time points for 16-bit Z-stacks, using an Image J custom macro. We calculated the average optical density $\left(\mathrm{AOD}_{\mathrm{Cy} 5}=\mathrm{RID}_{\mathrm{Cy} 5} / \mathrm{Area}_{\mathrm{ROI}} \mathrm{Cy}_{5}\right)$ for the optical slice that showed the maximum RID at 4 min post-injection. To account for any delay in the initiation of the acquisition protocol, we calculated the increase in $A \mathrm{DD}_{\mathrm{Cy} 5}$ from $t 1$ to $t 3$. To correct for variations in the quantity of solution injected, we similarly calculated the $A O D_{T M R}$ for an $\mathrm{ROI}_{\mathrm{TMR}}$ selected from the TMR stack acquired at 40x, for the optical slice with the maximum RID TMR. To correct for differences in the development stage of the injected oocytes, we measured the oocyte length from the anterior to the posterior of each injected egg chamber, and calculated a correction factor $\left(f_{\mathrm{A} \rightarrow \mathrm{P}}\right)$ by dividing this distance by the minimum distance of all measured oocytes. The final relative detection efficiency of each injection was calculated using Equation 2. The images shown in Fig. 8 are for the microinjections that presented the highest target detection efficiency for each molecular beacon, and the white rectangles highlight the ROls used to quantify each microinjection.

$$
\text { Relative efficiency }=\frac{\text {AOD }_{\text {ROI_Cy5 }}}{A O D_{\text {ROI_TMR }}} \times f_{A \rightarrow P}
$$

\section{SUPPLEMENTAL MATERIAL}

Supplemental material is available for this article. 


\section{ACKNOWLEDGMENTS}

We thank Ruth Lehman (Skirball Institute of Biomolecular Medicine, NYU School of Medicine) for the plasmid encoding full-length oskar mRNA. We thank Girish Ramrattan for useful discussions and advice on writing the PinMol software, and David Mathews (School of Medicine and Dentistry, University of Rochester Medical Center) for his advice and guidance with the RNAstructure package. We also thank Salvatore A.E. Marras (Public Health Research Institute Center, Rutgers University) for the synthesis of osk82 and osk2216 molecular beacons, for the labeling and purification of the osk82, osk2216, and osk1236 molecular beacons, and for his edits, suggestions, and comments on this manuscript. This work was supported by a National Science Foundation CAREER award 1149738 and a Professional Staff Congress-CUNY award to D.P.B.

Received November 9, 2018; accepted December 4, 2018.

\section{REFERENCES}

Bayer LV, Batish M, Formel SK, Bratu DP. 2015. Single-molecule RNA in situ hybridization (smFISH) and immunofluorescence (IF) in the Drosophila egg chamber. Methods Mol Biol 1328: 125-136. doi:10.1007/978-1-4939-2851-4_9

Bratu DP, Cha BJ, Mhlanga MM, Kramer FR, Tyagi S. 2003. Visualizing the distribution and transport of mRNAs in living cells. Proc Natl Acad Sci 100: 13308-13313. doi:10.1073/pnas.2233244100

Bratu DP, Catrina IE, Marras SA. 2011. Tiny molecular beacons for in vivo mRNA detection. Methods Mol Biol 714: 141-157. doi: 10.1007/978-1-61779-005-8_9

Catrina IE, Marras SA, Bratu DP. 2012. Tiny molecular beacons: LNA/ 2'-O-methyl RNA chimeric probes for imaging dynamic mRNA processes in living cells. ACS Chem Biol 7: 1586-1595. doi: 10.1021/cb300178a

Catrina IE, Bayer LV, Yanez G, McLaughlin JM, Malaczek K, Bagaeva E, Marras SA, Bratu DP. 2016. The temporally controlled expression of Drongo, the fruitfly homolog of AGFG1, is achieved in female germline cells via P-bodies and its localization requires functional Rab11. RNA Biol 13: 1117-1132. doi:10.1080/15476286.2016.1218592

Chen AK, Behlke MA, Tsourkas A. 2007. Avoiding false-positive signals with nuclease-vulnerable molecular beacons in single living cells. Nucleic Acids Res 35: e105. doi:10.1093/nar/gkm593

Chen AK, Rhee WJ, Bao G, Tsourkas A. 2011. Delivery of molecular beacons for live-cell imaging and analysis of RNA. Methods Mol Biol 714: 159-174. doi:10.1007/978-1-61779-005-8_10

Ephrussi A, Dickinson LK, Lehmann R. 1991. oskarorganizes the germ plasm and directs localization of the posterior determinant nanos. Cell 66: 37-50. doi:10.1016/0092-8674(91)90137-N

Griffiths-Jones S, Saini HK, van Dongen S, Enright AJ. 2008. miRBase: tools for microRNA genomics. Nucleic Acids Res 36: D154-D158. doi:10.1093/nar/gkm952

He Z, Wu L, Li X, Fields MW, Zhou J. 2005. Empirical establishment of oligonucleotide probe design criteria. Appl Environ Microbiol 71: 3753-3760. doi:10.1128/AEM.71.7.3753-3760.2005

Jackson SR, Wong AC, Travis AR, Catrina IE, Bratu DP, Wright DW, Jayagopal A. 2016. Applications of hairpin DNA-functionalized gold nanoparticles for imaging mRNA in living cells. Methods Enzymol 572: 87-103. doi:10.1016/bs.mie.2016.03.019

Kim-Ha J, Smith JL, Macdonald PM. 1991. oskarmRNA is localized to the posterior pole of the Drosophila oocyte. Cell 66: 23-35. doi:10.1016/0092-8674(91)90136-M
Lasko P. 2012. mRNA localization and translational control in Drosophila oogenesis. Cold Spring Harb Perspect Biol 4. doi:10.1101/ cshperspect.a012294

Lewis BP, Burge CB, Bartel DP. 2005. Conserved seed pairing, often flanked by adenosines, indicates that thousands of human genes are microRNA targets. Cell 120: 15-20. doi:10.1016/j.cell.2004. 12.035

Lorenz R, Bernhart SH, Höner zu Siederdissen C, Tafer H, Flamm C, Stadler PF, Hofacker IL. 2011. ViennaRNA Package 2.0. Algorithms Mol Biol 6: 26. doi:10.1186/1748-7188-6-26

Lu ZJ, Mathews DH. 2008a. Efficient siRNA selection using hybridization thermodynamics. Nucleic Acids Res 36: 640-647.

Lu ZJ, Mathews DH. 2008b. OligoWalk: an online siRNA design tool utilizing hybridization thermodynamics. Nucleic Acids Res 36: W104-W108. doi:10.1093/nar/gkn250

Marras SA, Kramer FR, Tyagi S. 2002. Efficiencies of fluorescence resonance energy transfer and contact-mediated quenching in oligonucleotide probes. Nucleic Acids Res 30: e122. doi:10.1093/nar/ gnf121

Mathews DH. 2006. RNA secondary structure analysis using RNAstructure. Curr Protoc Bioinformatics Chapter 12: Unit 12.6. doi:10.1002/0471250953.bi1206s13

Mathews DH, Disney MD, Childs JL, Schroeder SJ, Zuker M, Turner DH. 2004. Incorporating chemical modification constraints into a dynamic programming algorithm for prediction of RNA secondary structure. Proc Natl Acad Sci 101: 7287-7292. doi: 10.1073/pnas.0401799101

McLaughlin JM, Bratu DP. 2015. Drosophila melanogaster oogenesis: an overview. Methods Mol Biol 1328: 1-20. doi:10.1007/978-14939-2851-4_1

Mhlanga MM, Bratu DP, Genovesio A, Rybarska A, Chenouard N, Nehrbass U, Olivo-Marin JC. 2009. In vivo colocalisation of oskar mRNA and trans-acting proteins revealed by quantitative imaging of the Drosophila oocyte. PLoS One 4: e6241. doi:10.1371/journal.pone.0006241

Nazarenko I, Pires R, Lowe B, Obaidy M, Rashtchian A. 2002. Effect of primary and secondary structure of oligodeoxyribonucleotides on the fluorescent properties of conjugated dyes. Nucleic Acids Res 30: 2089-2195. doi:10.1093/nar/30.9.2089

Reuter JS, Mathews DH. 2010. RNAstructure: software for RNA secondary structure prediction and analysis. BMC Bioinformatics 11: 129. doi:10.1186/1471-2105-11-129

Rouskin S, Zubradt M, Washietl S, Kellis M, Weissman JS. 2014. Genome-wide probing of RNA structure reveals active unfolding of mRNA structures in vivo. Nature 505: 701-705. doi:10.1038/ nature12894

Schindelin J, Arganda-Carreras I, Frise E, Kaynig V, Longair M, Pietzsch T, Preibisch S, Rueden C, Saalfeld S, Schmid B, et al. 2012. Fiji: an open-source platform for biological-image analysis. Nat Methods 9: 676-682. doi:10.1038/nmeth.2019

Tsourkas A, Behlke MA, Bao G. 2002. Hybridization of 2'-O-methyl and 2'-deoxy molecular beacons to RNA and DNA targets. Nucleic Acids Res 30: 5168-5174. doi:10.1093/nar/gkf635

Tyagi S, Bratu DP, Kramer FR. 1998. Multicolor molecular beacons for allele discrimination. Nat Biotechnol 16: 49-53. doi:10.1038/ nbt0198-49

Weil TT, Parton RM, Davis I. 2010. Making the message clear: visualizing mRNA localization. Trends in Cell Biol 20: 380-390. doi: 10.1016/j.tcb.2010.03.006

Zuker M. 2003. Mfold web server for nucleic acid folding and hybridization prediction. Nucleic Acids Res 31: 3406-3415. doi:10.1093/ nar/gkg595 

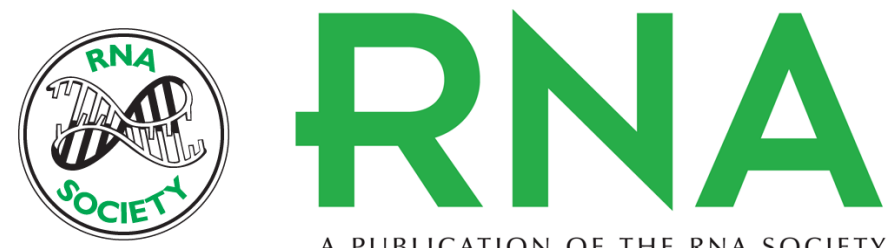

A PUBLICATION OF THE RNA SOCIETY

\section{PinMol: Python application for designing molecular beacons for live cell imaging of endogenous mRNAs}

Livia V. Bayer, Omar S. Omar, Diana P. Bratu, et al.

RNA 2019 25: 305-318 originally published online December 20, 2018

Access the most recent version at doi:10.1261/rna.069542.118

\section{Supplemental http://rnajournal.cshlp.org/content/suppl/2018/12/20/rna.069542.118.DC1 \\ Material}

References This article cites 30 articles, 4 of which can be accessed free at: http://rnajournal.cshlp.org/content/25/3/305.full.html\#ref-list-1

Creative This article is distributed exclusively by the RNA Society for the first 12 months after the Commons

License full-issue publication date (see http://rnajournal.cshlp.org/site/misc/terms.xhtml). After 12 months, it is available under a Creative Commons License (Attribution-NonCommercial 4.0 International), as described at http://creativecommons.org/licenses/by-nc/4.0/.

Email Alerting
Service

Receive free email alerts when new articles cite this article - sign up in the box at the top right corner of the article or click here.

\section{|||||||| Providing Precise Solutions for} your research.

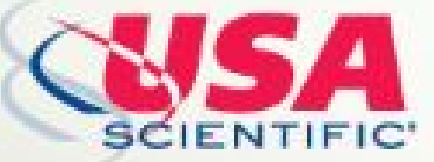

To subscribe to $R N A$ go to:

http://rnajournal.cshlp.org/subscriptions

(C) 2019 Bayer et al.; Published by Cold Spring Harbor Laboratory Press for the RNA Society 